\title{
Parallelotopes of Maximum Volume in a Simplex
}

\author{
M. Lassak \\ Instytut Matematyki i Fizyki ATR, \\ Kaliskiego 7, 85-796 Bydgoszcz, Poland \\ lassak@atr.bydgoszcz.pl
}

\begin{abstract}
It is proved that the maximum possible volume of a parallelotope contained in a $d$-dimensional simplex $S$ is equal to $\left(d ! / d^{d}\right) \operatorname{vol}(S)$. A description of all the parallelotopes of maximum volume contained in $S$ is given.
\end{abstract}

\section{Introduction}

Let $S$ be a nondegenerate simplex in Euclidean $d$-dimensional space $E^{d}$. Denote by $a$ a vertex of $S$ and by $v_{1}, \ldots, v_{d}$ the vectors determining the edges of $S$ at $a$. The parallelotope $P_{a}$ with vertex $a$ and the edges at $a$ determined by vectors $(1 / d) v_{1}, \ldots,(1 / d) v_{d}$ is a subset of $S$ and has volume $d ! / d^{d}$ times the volume of $S$. Our intuition says that there is no parallelotope contained in $S$ of greater volume. This conjecture is confirmed in Section 1. In Section 2 we describe all parallelotopes of maximum volume contained in $S$. We also show that all those parallelotopes are inscribed in $S$. Section 3 discusses the motivation of the present research and comments on some analogous results and problems, in particular, the problem of finding large simplices in a cube.

We denote by $\operatorname{aff}(K)$ and by $\operatorname{conv}(K)$ the affine and the convex hull of a set $K \subset E^{d}$. The symbol $\operatorname{vol}(C)$ denotes the volume of a convex body $C \subset E^{d}$. By $k C$ we mean the homothetic copy of $C$ of ratio $k$ and homothety center at the center of gravity of $C$.

Two sets in $E^{d}$ are said to be affinely equivalent if one of them is an image of the other under an affine nondegenerate transformation.

\section{The Maximum Volume of a Parallelotope Contained in a Simplex}

By a cylinder parallel to a direction $\delta$ we mean the union of a family of segments parallel to $\delta$ whose endpoints are in two different parallel hyperplanes. The segments are called 
$\delta$-segments of the cylinder. If the above two hyperplanes are perpendicular to $\delta$, then the cylinder is called rectangular. The smallest rectangular cylinder parallel to $\delta$ and containing a given compact set $X$ is denoted by $\operatorname{cyl}(X, \delta)$.

Lemma 1. Let $S \subset E^{d}$ be a nondegenerate simplex and let $Y \subset E^{d}$ be a cylinder parallel to a direction $\delta$. If $-(1 / d) S \subset Y \subset S$, then the simplex $-(1 / d) S$ contains exactly one $\delta$-segment from among the $\delta$-segments of $Y$.

Proof. Since for every cylinder there exists an affinely equivalent rectangular cylinder, we limit our considerations to the case when $Y$ is a rectangular cylinder.

From the definition of $\operatorname{cyl}(X, \delta)$ we see that, in order to show the existence of a $\delta$ segment of $Y$ contained in $-(1 / d) S$, it is sufficient to show this in the special case of $\operatorname{cyl}(-(1 / d) S, \delta)$ in place of $Y$. Thus let $Y=\operatorname{cyl}(-(1 / d) S, \delta)$ from now on. Denote by $G$ and $H$ the two hyperplanes supporting $Y$ which are perpendicular to $\delta$. Observe that each of the hyperplanes $G$ and $H$ contains at least one vertex of the simplex $-(1 / d) S$.

We consider three cases in order to prove the existence of a $\delta$-segment of $Y$ in $-(1 / d) S$.

Case 1: exactly one vertex of $-(1 / d) S$ is in $G$ and exactly $d$ vertices of $-(1 / d) S$ are in $H$ (or vice-versa). Denote by $g$ this vertex of $-(1 / d) S$ which is in $G$, and by $F$ the opposite face of $-(1 / d) S$. Since $d$ vertices of $F$ are in $H$, we see that $F \subset H$. Let $F^{\prime}$ be the perpendicular projection of $F$ on $G$. Obviously, $F^{\prime}$ is a homothetic copy of $S \cap G$. The homothety ratio is $-1 / d$. Since $g$ is the center of the simplex $S \cap G$, every $(d-2)$-dimensional plane parallel to a $(d-2)$-dimensional face of $S \cap G$ and passing through $g$ is $d$ times closer to this face than to the opposite vertex of $S \cap G$. We conclude that $F^{\prime}$ has nonempty intersections with all the $(d-2)$-dimensional planes through $g$ which are parallel to the $(d-2)$-dimensional faces of $F^{\prime}$. Thus $g \in F^{\prime}$ and $Y=\operatorname{conv}\left(F \cup F^{\prime}\right)$. Consequently, the $\delta$-segment of cylinder $Y$ with endpoint $g$ is the $\delta$-segment we are looking for.

Case 2: all vertices of $-(1 / d) S$ are in $G \cup H$, and each of the hyperplanes $G$ and $H$ contains more than one vertex of $-(1 / d) S$. We apply the indirect approach: we assume that $-(1 / d) S$ contains no $\delta$-segment of $Y$ and we show that $Y$ is not a subset of $S$.

Denote by $g_{1}, \ldots, g_{m}$ those vertices of $-(1 / d) S$ which are in $G$, and by $h_{1}, \ldots, h_{n}$ the vertices of $-(1 / d) S$ which are in $H$. We have $2 \leq m \leq d-1$ and $2 \leq n \leq d-1$. Denote by $g_{i}^{\prime}$ the projection of $g_{i}$ on $H$, where $i=1, \ldots, m$, and denote by $h_{i}^{\prime}$ the (perpendicular) projection of $h_{i}$ on $G$, where $i=1, \ldots, n$ (see Fig. 1, where $d=3$ and $m=n=2$ ).

The planes aff $\left\{g_{1}^{\prime}, \ldots, g_{m}^{\prime}\right\}$ and $\operatorname{aff}\left\{h_{1}, \ldots, h_{n}\right\}$ are contained in the hyperplane $H$. Since $m+n=d+1$ and since the simplex $-(1 / d) S$ is nondegenerate, these planes have exactly one common point. Denote this point by $o$. Let $o^{\prime}$ denote the projection of $o$ on $G$. Our assumption that $-(1 / d) S$ contains no $\delta$-segment of $Y$ (remember that $Y=\operatorname{cyl}(-(1 / d) S, \delta))$ implies that $\operatorname{conv}\left\{h_{1}, \ldots, h_{m}\right\}$ or $\operatorname{conv}\left\{g_{1}^{\prime}, \ldots, g_{n}^{\prime}\right\}$ does not contain $o$. Assume the first possibility (for the second possibility further consideration is analogical).

Consider the $n$-dimensional plane $K$ containing $h_{1}, \ldots, h_{n}, h_{1}^{\prime}, \ldots, h_{n}^{\prime}, o, o^{\prime}$. Denote by $S^{*}$ the simplex such that $-(1 / n) S^{*}$ is the $n$-dimensional simplex with vertices $h_{1}, \ldots, h_{n}$ and $o^{\prime}$. Of course, $-(1 / n) S^{*} \subset S^{*} \subset K$. Let $\Pi$ denote the projection of the 


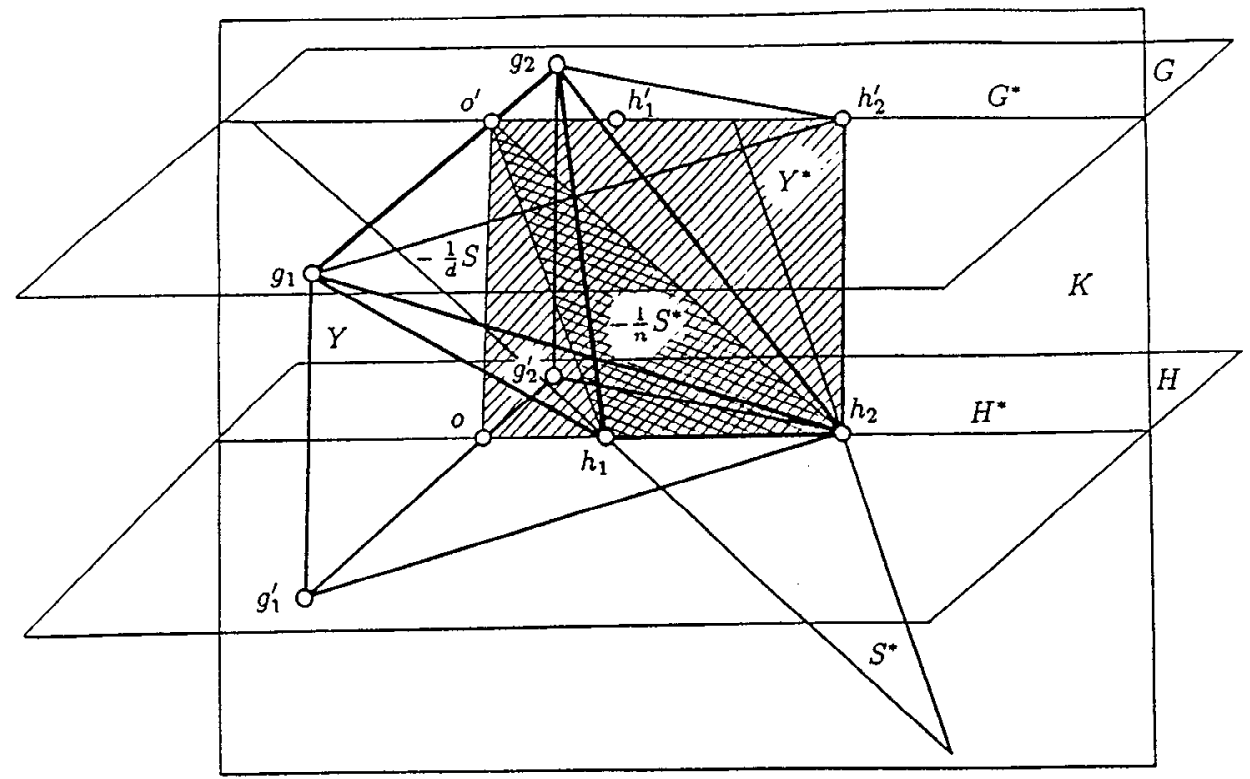

Fig. 1

space $E^{d}$ on the plane $K$ such that $\Pi\left(\operatorname{aff}\left\{g_{1}^{\prime}, \ldots, g_{m}^{\prime}\right\}\right)=\{o\}$. We have $\Pi(S)=S^{*}$ and $\Pi(-(1 / d) S)=-(1 / n) S^{*}$. We apply the $n$-dimensional version of Case 1 to simplices $-(1 / n) S^{*}$ and $S^{*}$. In place of $G$ (as in Case 1$)$ we take the $(n-1)$-dimensional plane $G^{*}=\operatorname{aff}\left\{h_{1}^{\prime}, \ldots, h_{n}^{\prime}\right\}$. The role of $H$ (as in Case 1$)$ is played by $H^{*}=\operatorname{aff}\left\{h_{1}, \ldots h_{n}\right\}$. Since $o \notin \operatorname{conv}\left\{h_{1}, \ldots, h_{n}\right\}$, we see that $-(1 / n) S^{*}$ contains no $\delta$-segment of the cylin$\operatorname{der} Y^{*}=\operatorname{cyl}\left(-(1 / n) S^{*}, \delta\right)$. Applying the $n$-dimensional version of Case 1 we conclude that $S^{*}$ does not contain $Y^{*}$. Thus an $(n-1)$-dimensional face of $S^{*}$ does not support $Y^{*}$. Since the $(n-1)$-dimensional face of $-(1 / n) S^{*}$ opposite the vertex $o^{\prime}$ lies in $H^{*}$, we conclude that the parallel $(n-1)$-dimensional face of $S^{*}$ supports $Y^{*}$ at $o^{\prime}$. Thus there is a vertex $h_{i}$, where $i \in\{1, \ldots, n\}$, such that the hyperplane carrying the $(n-1)$ dimensional face of $S^{*}$ containing $h_{i}$ does not support $Y^{*}$ (in Fig. 1 both $h_{1}$ and $h_{2}$ illustrate $h_{i}$ ). This and $\Pi(Y)=Y^{*}$ imply that the plane carrying the facet of $S$ passing through $h_{i}$ does not support $Y$. Thus $S$ does not contain $Y$.

Case 3: at least one vertex of $-(1 / d) S$ is out of $G \cup H$. Denote by $u_{0}, \ldots, u_{k}$ those vertices of $-(1 / d) S$ which are in $G \cup H$ and denote the remaining vertices by $u_{k+1}, \ldots, u_{d}$ (Fig. 2 shows the case when $d=3$ and $k=2$ ). Let $V=\operatorname{aff}\left\{u_{0}, \ldots, u_{k}\right\}$. Denote by $S^{\prime}$ the $k$-dimensional simplex such that $-(1 / k) S^{\prime}=\operatorname{conv}\left\{u_{0}, \ldots, u_{k}\right\}$. Of course, $-(1 / k) S^{\prime} \subset S^{\prime} \subset V$ and $-(1 / k) S^{\prime}=V \cap-(1 / d) S$.

Since $-(1 / d) S \subset Y \subset S$ and since the vertices of $-(1 / d) S$ are in the boundary of $S$, we see that they are boundary points of $Y$. Thus from the fact that $u_{k+1}, \ldots, u_{d}$ are not in $G \cup H$ and that $Y=\operatorname{cyl}(-(1 / d) S, \delta)$ we conclude that $\delta$ is parallel to all those $d-k$ facets of $S$ which contain $u_{k+1}, \ldots, u_{d}$. Hence $\delta$ is parallel to all the $d-k$ 


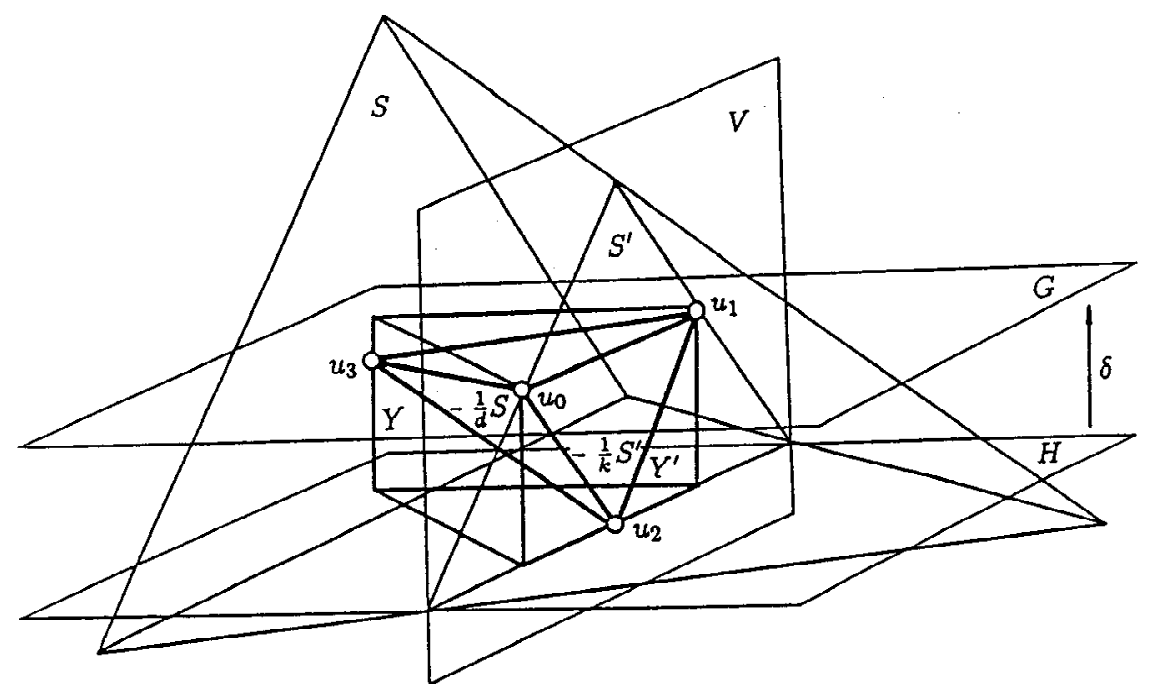

Fig. 2

facets of $-(1 / d) S$ that are opposite those vertices. Consequently, $\delta$ is parallel to $V$. Thus $V \cap Y=Y^{\prime}$, where $Y^{\prime}=\operatorname{cyl}\left(-(1 / k) S^{\prime}, \delta\right)$.

Observe that

$$
-\frac{1}{k} S^{\prime} \subset Y^{\prime} \subset S^{\prime}
$$

The left inclusion is obvious. Since every $(d-1)$-dimensional face of $S$ supports $Y$ at a vertex of $-(1 / d) S$, each $(k-1)$-dimensional face of $S^{\prime}$ supports $Y^{\prime}$ at a vertex of $-(1 / k) S$. Hence the right inclusion also holds.

We take into account (1) and apply the $k$-dimensional version of Case 2, where $V \cap G$ and $V \cap H$ play the parts of $G$ and $H$ (as in Case 2), respectively. We conclude that a $\delta$ segment of cylinder $Y^{\prime}$ is contained in the simplex $-(1 / k) S^{\prime}$. Obviously, this $\delta$-segment is contained in $Y$ as well, and thus is the $\delta$-segment that we are looking for.

This settles Case 3.

We have thus shown the existence of a $\delta$-segment of $Y$ in the simplex $-(1 / d) S$.

The promised uniqueness of the $\delta$-segment is a consequence of the following property of a $d$-dimensional simplex: the sum of dimensions of two faces of a simplex being intersections of this simplex with two opposite supporting hyperplanes is at most $d-1$. It is enough to consider the projection of one of the hyperplanes onto the other.

From Lemma 1 we immediately obtain the following lemma.

Lemma 2. Let $S \subset E^{d}$ be a simplex, let $P \subset E^{d}$ be a nondegenerate parallelotope, and let $\delta_{1}, \ldots, \delta_{d}$ be the directions of the edges of $P$. If $-(1 / d) S \subset P \subset S$, then the simplex $-(1 / d) S$ contains precisely one $\delta_{i}$-segment $A_{i}$ of $P$ for every $i \in\{1, \ldots, d\}$. 


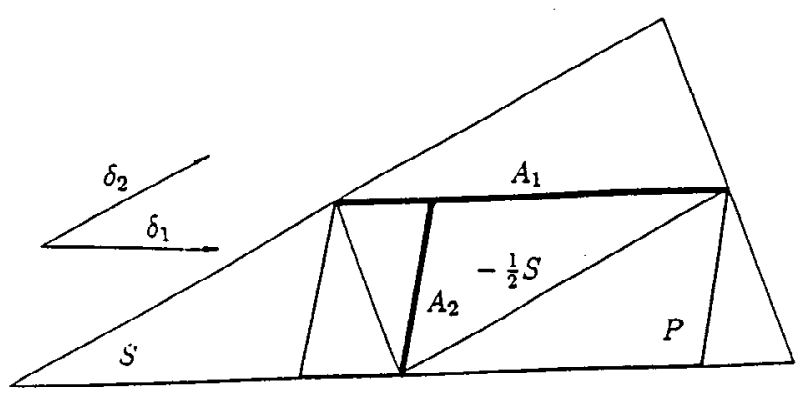

Fig. 3

Figure 3 shows a planar illustration of Lemma 2, and three-dimensional illustrations are given in Figs. 4 and 5.

Let the assumptions of Lemma 2 hold for a parallelotope $P$ and a simplex $S$. Then we call $A_{1}, \ldots, A_{d}$ the segments connecting the opposite facets of $P$, or the connecting segments for short. Denote by $o$ a vertex of $P$. For every $i \in\{1, \ldots, d\}$ we denote by $B_{i}$ the edge of $P$ which is a translate of $A_{i}$ and whose endpoint is $o$. Moreover, for convenience of further considerations, we introduce a coordinate system such that $o$ is the coordinate center, and such that the $i$ th coordinate axis contains $B_{i}$ in its positive half, where $i=1, \ldots, d$.

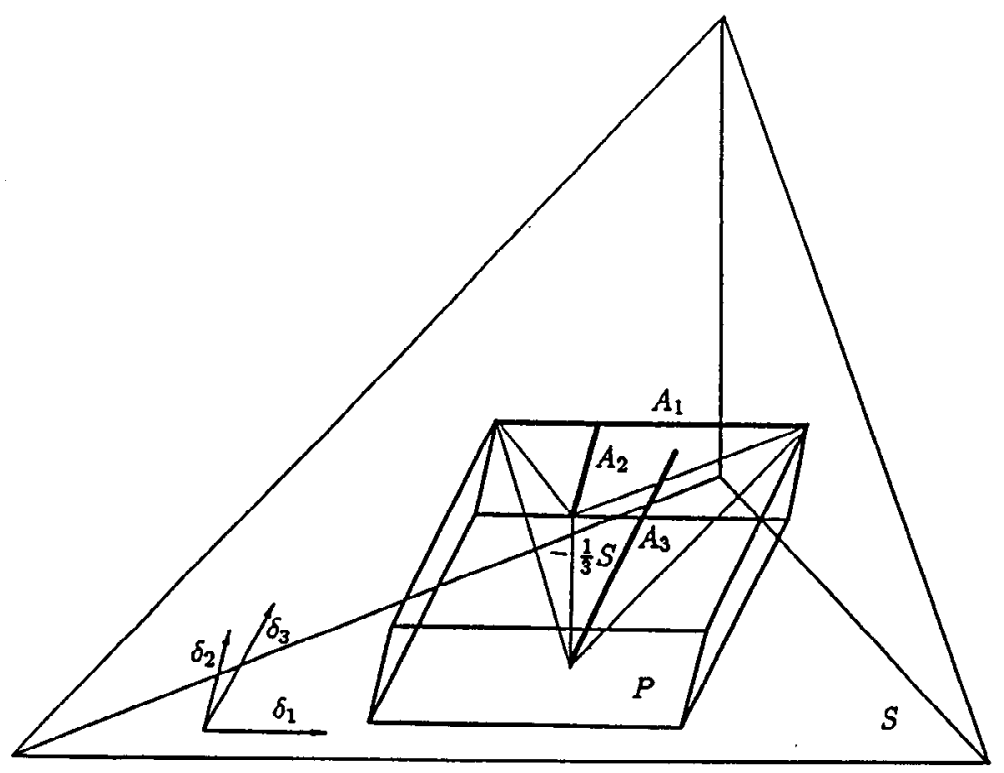

Fig. 4 


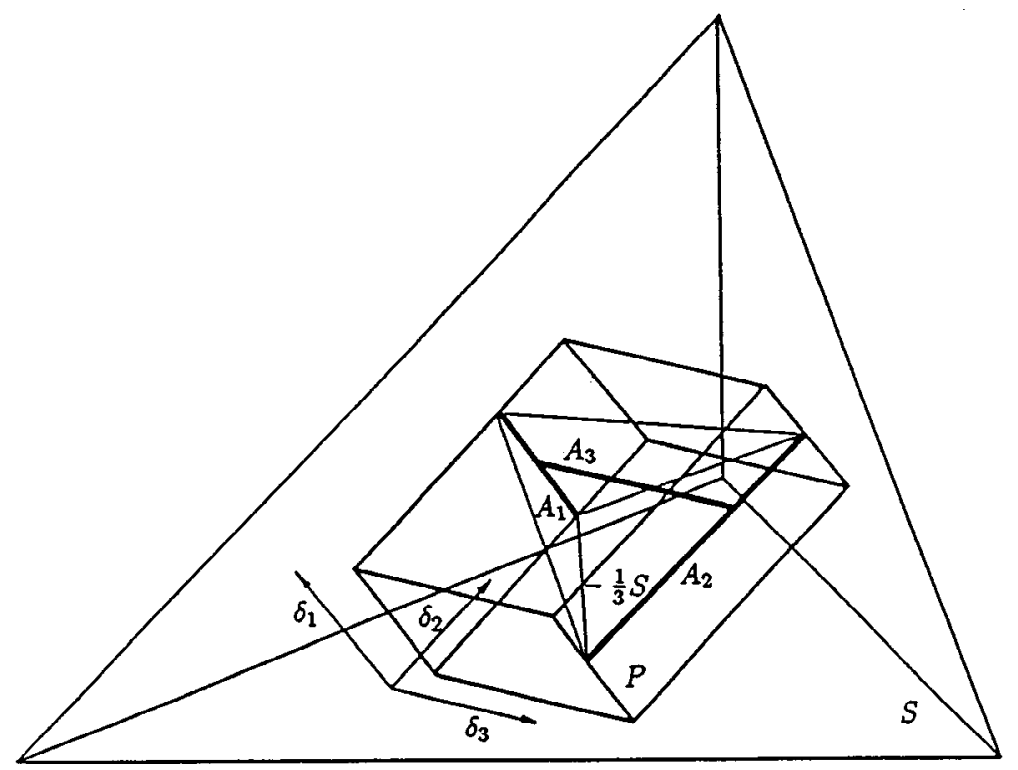

Fig. 5

Obviously, we have

$$
\operatorname{vol}(P)=d ! \cdot \operatorname{vol}\left[\operatorname{conv}\left(\bigcup_{i=1}^{d} B_{i}\right)\right]
$$

Every convex body $C$ can be represented as the union of segments parallel to the $i$ th axis, where $i \in\{1, \ldots, d\}$. We translate these segments parallel to the $i$ th axis in order to get them in the positive half-space $x_{i} \geq 0$ such that an endpoint of each segment is contained in the hyperplane $x_{i}=0$. The union of the translated segments is again a convex body and its volume remains unchanged (see [14]). We denote the obtained body by $\rho_{i}(C)$. This is Radziszewski's operation introduced in [14]. Consider the superposition $\rho$ of operations $\rho_{1}, \ldots, \rho_{d}$. Clearly, $\rho$ transforms each convex body into a convex body of the same volume.

Observe that the set $\rho\left[\operatorname{conv}\left(\bigcup_{i=1}^{d} A_{i}\right)\right]$ contains $B_{1}, \ldots, B_{d}$. Since this set is convex, it contains conv $\left(\bigcup_{i=1}^{d} B_{i}\right)$ as well. Since $\rho$ does not change the volume of a convex body, we obtain

$$
\operatorname{vol}\left[\operatorname{conv}\left(\bigcup_{i=1}^{d} B_{i}\right)\right] \leq \operatorname{vol}\left[\operatorname{conv}\left(\bigcup_{i=1}^{d} A_{i}\right)\right] .
$$

We conjecture that $\operatorname{vol}\left[\operatorname{conv}\left(\bigcup_{i=1}^{d} A_{i}\right)\right]=(1 / d !) \operatorname{vol}(P)$.

Lemma 3. If $P$ is a parallelotope of maximum volume contained in a d-dimensional simplex $S$, then $-(1 / d) S \subset P$. 


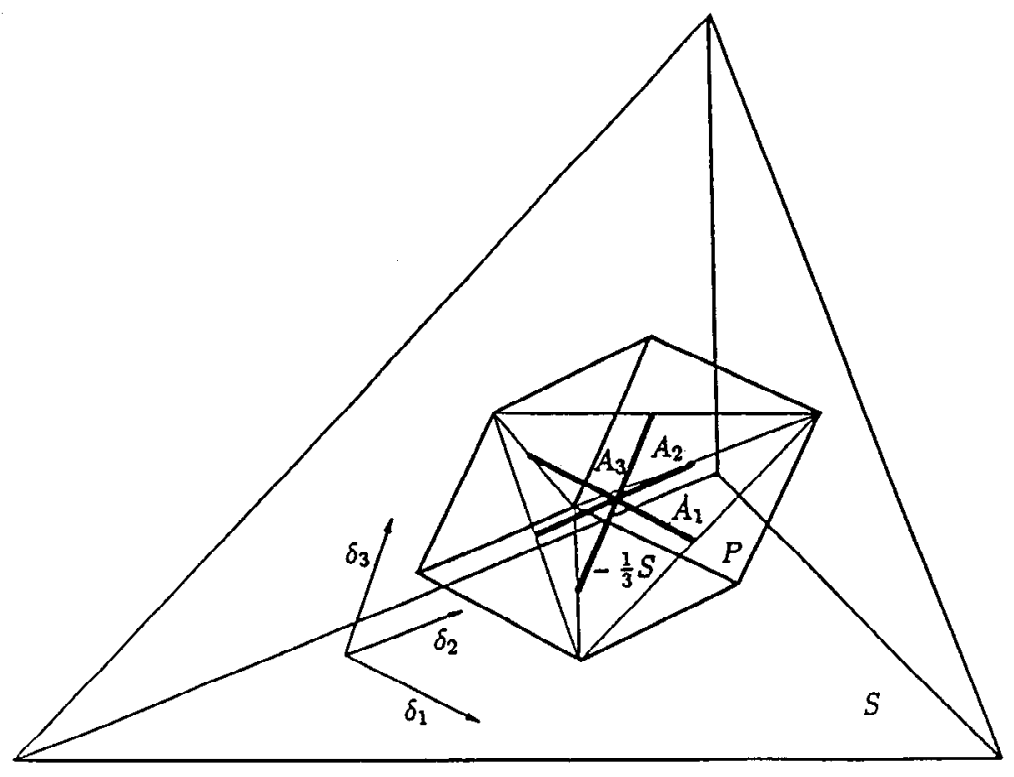

Fig. 6

Proof. From [8] we know that a $d$ times larger homothetic copy $R$ of $P$ contains $S$. Since $R$ is centrally symmetric, it also contains a translate of $-S$. Consequently, $P$ contains a translate of $-(1 / d) S$. Since $P \subset S$, this translate is contained in $S$. Thus it is nothing other than $-(1 / d) S$ itself.

Lemma 3, and also Lemma 4 and Theorem 1 below, are illustrated in Fig. 3 for $d=2$, and in Figs. 4 and 5 for $d=3$ Observe that Fig. 6 does not illustrate Lemmas 3 and 4 and Theorem 1 (but it does illustrate Lemma 2). The reason is that the parallelotope in Fig. 6 is not a parallelotope of maximum volume contained in the simplex. We see that the condition $-(1 / d) S \subset P \subset S$ does not characterize parallelotopes of maximum volume contained in $S$.

Lemma 4. If $P$ is a parallelotope of maximum volume contained in a d-dimensional simplex $S$, then $-(1 / d) S=\operatorname{conv}\left(\bigcup_{i=1}^{d} A_{i}\right)$.

Proof. As explained at the beginning of this paper, $S$ contains a parallelotope of volume $\left(d ! / d^{d}\right) \operatorname{vol}(S)$. By the assumed maximality of the volume of $P$ we obtain that $\operatorname{vol}(P) \geq$ $\left(d ! / d^{d}\right) \operatorname{vol}(S)$. Hence

$$
\operatorname{vol}\left(-\frac{1}{d} S\right) \leq \frac{1}{d !} \operatorname{vol}(P)
$$

Because of Lemma 3 we can use Lemma 2 which permits us to apply (2) and (3). Moreover, we apply (4). We obtain $\operatorname{vol}(-(1 / d) S) \leq \operatorname{vol}\left[\operatorname{conv}\left(\bigcup_{i=1}^{d} A_{i}\right)\right]$. This 
inequality and the inclusion $\operatorname{conv}\left(\bigcup_{i=1}^{d} A_{i}\right) \subset-(1 / d) S$ imply that $-(1 / d) S$ $=\operatorname{conv}\left(\bigcup_{i=1}^{d} A_{i}\right)$.

The author conjectures that the inverse implication to this in Lemma 4 holds true as well, i.e., that the equality $-(1 / d) S=\operatorname{conv}\left(\bigcup_{i=1}^{d} A_{i}\right)$ characterizes every parallelotope $P$ of maximum volume contained in $S$; of course, under the assumption that $-(1 / d) S \subset$ $P \subset S$.

Theorem 1. If $P$ is a parallelotope of maximum volume in a $d$-dimensional simplex $S$, then $\operatorname{vol}(P)=\left(d ! / d^{d}\right) \operatorname{vol}(S)$.

Proof. We apply Lemma 4. Moreover, owing to Lemmas 2 and 3, we apply (2) and (3). We see that $\operatorname{vol}(-(1 / d) S) \geq(1 / d !) \operatorname{vol}(P)$. This inequality and (4) mean that Theorem 1 holds true.

Corollary. If $S$ is a simplex of minimum volume containing a parallelotope $P$, then $\operatorname{vol}(S)=\left(d^{d} / d !\right) \operatorname{vol}(P)$.

In connection with Theorem 1 it is natural to ask what is the maximum volume of a zonotope contained in a $d$-dimensional simplex $S$ (or, equivalently, in a regular simplex). It is easy to see that if $d=2$, then this maximum volume, $\frac{2}{3} \operatorname{vol}(S)$, is attained for the zonotope $S \cap(-S)$. The author conjectures that for $d=3$ this maximum volume is $\frac{4}{9} \operatorname{vol}(S)$. The corresponding zonotope is obtained from the octahedron $S \cap(-S)$ by truncating prisms at its vertices by hyperplanes parallel to pairs of opposite edges of $S$ such that one-third of each edge of the octahedron is cut off. The question seems to be more difficult if an analogical construction for higher dimensions also gives a zonotope of maximum volume in $S$. Paper [5] considers centrally symmetric convex bodies of the maximum volume contained in a simplex.

A natural conjecture concerning Theorem 1 would be that for every convex body $C \subset E^{d}$ there is a parallelotope $P \subset C$ such that $\operatorname{vol}(P) \geq\left(d ! / d^{d}\right) \operatorname{vol}(C)$. Surprisingly, such a conjecture is not true in general (see [1]). We only know that it is true for $d=2$ and $d=3$, see [14] and [2], and that there exists a parallelotope in $C$ whose volume is at least $d^{-d+1} \operatorname{vol}(C)$, see [10] (this is a slight improvement on the estimate $d^{-d} \operatorname{vol}(C)$ from [12]).

We pose a problem about the generalization of Theorem 1: evaluate the maximum $j$ dimensional volume of a $j$-dimensional parallelotope in a regular $d$-dimensional simplex and describe all those largest $j$-dimensional parallelotopes. Of course, the largest onedimensional parallelotopes are the edges of the regular simplex. The author conjectures that, for $d \geq 3$, the maximum two-dimensional volume of a two-dimensional parallelotope (i.e., the maximum area of a parallelogram) in a regular $d$-simplex of edge length 1 is $\frac{1}{4}$. If $d=3$, then this value is attained for the convex hull of two segments, each of which connects the midpoints of the opposite edges of the simplex. For $d \geq 4$, this value is attained for the above described parallelograms contained in the three-dimensional faces of the simplex. 


\section{Description of the Parallelotopes of Maximum Volume Contained in a Simplex}

A sequence $\Phi$ of $d$ segments $S_{1}, \ldots, S_{d}$, called frame-segments, contained in a $d$ dimensional simplex $R$ is said to be a frame of $R$ if the following frame-construction, consisting of $d+1$ stages, is possible. At the beginning, i.e., at stage 0 , we have 0 frame-segments. We also have $d+1$ so-called frame-faces; they are the vertices of $R$. When passing from the $(k-1)$ st stage to the $k$ th stage, we add segment $S_{k}$. It should connect two disjoint frame-faces. Simultaneously, these two frame-faces are removed and one new frame-face is added. This new frame-face is the convex hull of the union of the removed ones. Clearly, at the $j$ th stage, where $j \in\{0, \ldots, d\}$, we have $j$ framesegments and $d-j+1$ frame-faces. Thus at the $d$ th stage we have $d$ frame-segments and one frame-face $R$.

The two frame-faces at stage $d-1$ are called the main frame-faces. By the main frame-segment we mean the frame-segment $S_{d}$ added when passing to the $d$ th stage.

Figures 7-9 show frames of a three-dimensional simplex with vertices $a, b, c, d$. When passing to successive stages of the frame-construction, we successively add framesegments $a b, c e, d f$ (Fig. 7) and $a d, b c, g h$ (Fig. 8). Sometimes a number of different frame-constructions using the same segments is possible. In Fig. 9 we can make three different frame-constructions by choosing the following orders of using the frame segments: order $a b, a c, d k$, order $a c, a b, d k$, and order $a c, d k, a b$. In Fig. 8 one more order, $b c, a d, g h$, is also possible. In Fig. 7 only one order is possible.

If frame-construction is possible at least up to the $j$ th stage, where $j \in\{0, \ldots, d\}$, then at the $j$ th stage the following two obvious properties hold true.

(i) Every frame-face of dimension at least 1 is the convex hull of the frame-segments contained in it. The number of those frame-segments is equal to the dimension of the frame-face.

(ii) Every two different frame-faces at a fixed stage have empty intersection.

From the definition of the frame we conclude that vectors determined by the framesegments $S_{1}, \ldots, S_{d}$ are linearly independent.

By the parallelotope constructed over a frame we mean the smallest parallelotope

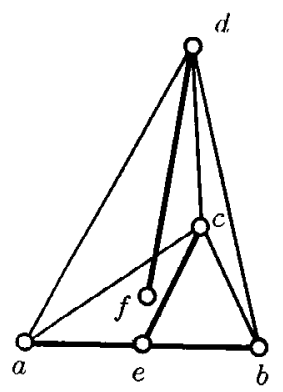

Fig. 7

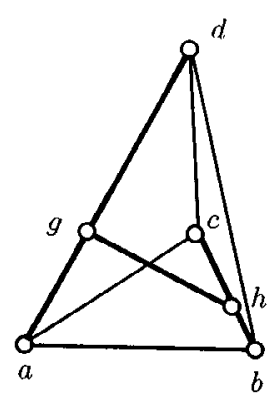

Fig. 8

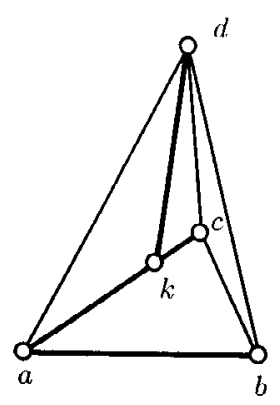

Fig. 9 
which contains this frame and whose edges are parallel to the frame-segments. Following are two properties of the parallelotopes constructed over frames.

(iii) Let $S_{1}, \ldots, S_{d}$ be a frame of a d-dimensional simplex $R$ and let $P$ be the parallelotope constructed over this frame. For every $k \in\{1, \ldots, d\}$, the pair of faces of $P$ parallel to all frame-segments different from $S_{k}$ contains the endpoints of segment $S_{k}$.

Assume the opposite. Then a face $F$ of $P$ parallel to all frame-segments different from $S_{k}$ contains no endpoint of $S_{k}$. Denote by $G$ the greatest (by inclusion) frame-face obtained during the process of frame-construction which is contained in $F$. Since $G$ contains no endpoint of $S_{k}$, all endpoints of the frame-segments not contained in $G$ are out of $G$. Since $G$ is smaller than $R$, we see that the frame-construction process cannot be provided to the end. A contradiction.

From (iii) we immediately obtain the following property.

(iv) The edges of the parallelotope constructed over a frame are translates of the frame-segments.

In Fig. 3 we see the parallelogram constructed over the frame $A_{1}, A_{2}$ of the simplex $-\frac{1}{2} S$. In Figs. 4 and 5 we have parallelotopes constructed over the frame $A_{1}, A_{2}, A_{3}$ of $-\frac{1}{3} S$. Figures 3-5 (but not Fig. 6) illustrate the following theorem.

Theorem 2. The parallelotopes of maximum volume in a $d$-dimensional simplex $S$ coincide with the parallelotopes constructed over the frames of the simplex $-(1 / d) S$. The parallelotopes are inscribed in $S$.

Proof. We divide the proof into three parts.

(I) We show that if $P$ is a parallelotope of maximum volume contained in $S$ and if $A_{1}, \ldots, A_{d}$ are the connecting segments (see Lemma 3, Lemma 2, and the definition just after), then we can construct a frame of $-(1 / d) S$ using $A_{1}, \ldots, A_{d}$ in place of the frame-segments.

Assume that our frame-construction has been performed up to the $j$ th stage, where $j \in\{0, \ldots, d-1\}$. We intend to show that passing to the $(j+1)$ st stage is possible.

We start by showing that, in each frame-face $K$, at the $j$ th stage there is an endpoint of a connecting segment different from the connecting segments contained in this frameface. Denote the dimension of $K$ by $k$. If $k=0$, i.e., if $K$ is a vertex of $S$, then from Lemma 4 we conclude that a connecting segment has an endpoint at $K$. Let $k \geq 1$. From (i) we see that $K$ is the convex hull of some $k$ connecting segments. To simplify the notation, assume that these segments are $A_{1}, \ldots, A_{k}$. Put $K=\operatorname{conv}\left(A_{1} \cup \cdots \cup A_{k}\right)$ and $L=\operatorname{conv}\left(A_{k+1} \cup \cdots \cup A_{d}\right)$. Assume that in $K$ there is no endpoint of a connecting segment different from the connecting segments contained in $K$. Observe that none of the segments $A_{1}, \ldots, A_{k}$ has an endpoint in $L$ and that none of the segments $A_{k+1}, \ldots, A_{d}$ has an endpoint in $K$ (the first statement results from the construction of a frame, and the second statement is nothing other than our assumption). This and the fact that the connecting segments are parallel to the edges of $P$ and that they connect pairs of opposite 
facets of $P$ imply that conv $(K \cup L)$ has at least $d+2$ vertices. This contradicts the equality (resulting from Lemma 4$)$ that $-(1 / d) S=\operatorname{conv}(K \cup L)$.

At the $j$ th stage we have $d-j+1$ frame-faces. They contain exactly $j$ connecting segments. From (ii) we see that every endpoint of each of the remaining $d-j$ connecting segments is contained in at most one of the above $d-j+1$ faces. Thus from the fact shown in the preceding paragraph we conclude that there is a pair of frame-faces containing the endpoints of a connecting segment. Thus we can pass to the $(j+1)$ st stage.

We see that a permutation of $\left\{A_{1}, \ldots, A_{d}\right\}$ is a frame of the simplex $-(1 / d) S$.

(II) By induction we show that the parallelotope constructed over an arbitrary frame of $-(1 / d) S$ is inscribed in $S$.

Of course, this property holds true in $E^{1}$. Assume that it holds true in $E^{1}, \ldots, E^{d-1}$ and consider the space $E^{d}$.

Let $S_{1}, \ldots, S_{d}$ be a frame $\Phi$ of $-(1 / d) S$. Let $S_{d}$ be the main frame-segment. Consider the frame $\Phi^{*}$ of $S$ consisting of the successive frame-segments $S_{i}^{*}$ that are images of the frame segments $S_{i}$, where $i=1, \ldots d$, under homothety with ratio $-d$ and center in the gravity center of $S$. Of course, $S_{d}^{*}$ is the main frame-segment of frame $\Phi^{*}$. Let $M_{1}$ and $M_{2}$ be the main frame-faces of frame $\Phi^{*}$. Let $m_{i}$ denote the number of frame-segments in $M_{i}$, where $i=1,2$. It is clear that $m_{1}+m_{2}=d-1$ and that $S_{d}^{*}$ has endpoints in $M_{1}$ and $M_{2}$.

Let $T$ be the parallelotope whose vertices are of the form $(1 / d) \sum_{k=1}^{d} s_{k}$, where $s_{k}$ is an endpoint of $S_{k}^{*}$ for $k=1, \ldots, d$. Observe that every edge of $T$ is a translate of a segment $S_{k}$, where $k \in\{1, \ldots, d\}$. From (iv) we see that the parallelotope $T$ contains a translate of the simplex $-(1 / d) S$. This, the obvious inclusion $T \subset S$, and the fact that $-(1 / d) S$ is the only homothetic image of $S$ of ratio $-1 / d$ that is contained in $S$, imply that $T$ is nothing other than the parallelotope constructed over frame $\Phi$ of $-(1 / d) S$.

In order to show that $T$ is inscribed in $S$, take an arbitrary vertex $w=(1 / d) \sum_{k=1}^{d} s_{k}$ of $T$. We intend to show that $w$ is on the boundary of $S$. Clearly, $m_{i}$ of points $s_{1}, \ldots, s_{d}$ are in $M_{i}$, where $i=1,2$. One among the points $s_{1}, \ldots, s_{d}$ is an endpoint of $S_{d}^{*}$. Thus it is in $M_{1}$ or in $M_{2}$. Without loss of generality we may assume that this endpoint is in $M_{1}$. We see that $m_{1}+1$ among the points $s_{1}, \ldots, s_{d}$ are in $M_{1}$, and that the remaining $m_{2}$ of these points are in $M_{2}$. Of course, if $m_{1}=d-1$, then $w$ is in the boundary of $S$. Consider the case when $m_{1} \leq d-2$. Now $m_{2} \geq 1$. Vertex $w$ is in a segment whose one endpoint $w_{1}$ is in the convex hull of the aforementioned $m_{1}+1$ points, and the second endpoint $w_{2}$ is in the convex hull of the remaining $m_{2}$ points. Of course, $w_{1} \in M_{1}$. Moreover, by the inductive assumption, $w_{2}$ is in the relative boundary of $M_{2}$. Consequently, $w$ is on the boundary of $S$. We see that $T$ is inscribed in $S$.

(III) We intend to prove that the parallelotope $T$ constructed over an arbitrary frame of $-(1 / d) S$ is a parallelotope of maximum volume contained in $S$. Because of the inclusion $T \subset S$ shown in (II) and because of Theorem 1, it is sufficient to show that

$$
\operatorname{vol}(T)=\frac{d !}{d^{d}} \operatorname{vol}(S)
$$

i.e., that $\operatorname{vol}(-(1 / d) S)=(1 / d !) \operatorname{vol}(T)$. Since every two $d$-dimensional parallelotopes are affinely equivalent, we do not make our considerations narrower assuming that $T$ is 
the unit cube. Consequently, we have to show that

$$
\operatorname{vol}\left(-\frac{1}{d} S\right)=\frac{1}{d !}
$$

We show this by induction.

Of course, (6) holds true in $E^{1}$.

Assume that (6) is true in $E^{1}, \ldots, E^{d-1}$ and consider the space $E^{d}$. By the definition of a frame we see that the main frame-segment connects two frame-faces of dimensions $r$ and $d-r-1$, where $r \in\{0, \ldots, d-1\}$. By the inductive assumption, the $r$-dimensional volume of the first frame-face is $1 / r$ !, and the $(d-r-1)$-dimensional volume of the second face is $1 /(d-r-1)$ !. Presenting $-(1 / d) S$ as the union of sections by hyperplanes perpendicular to the main frame-segment, we obtain

$$
\operatorname{vol}\left(-\frac{1}{d} S\right)=\int_{0}^{1} \frac{x^{r}}{r !} \frac{(1-x)^{d-r-1}}{(d-r-1) !} d x=\frac{1}{d !} .
$$

This gives (6) and thus confirms (5). Consequently, $T$ is a parallelotope of maximum possible volume in $S$.

Let $P$ be a parallelotope of the maximum volume contained in a simplex $S$. From the definition of a frame and from Theorem 2 we see how the vertices of $-(1 / d) S$ are distributed in the boundary of $P$. There is a pair $P(1), P(2)$ of opposite facets of $P$ which contains all vertices of $-(1 / d) S$ (see Figs. 3-5). If $P\left(j_{1}\right)$, where $j_{1} \in\{1,2\}$, contains more than one vertex of $-(1 / d) S$, then there is a pair $P\left(j_{1}, 1\right), P\left(j_{1}, 2\right)$ of opposite facets of $P\left(j_{1}\right)$, which contains all vertices of $-(1 / d) S$ lying in $P\left(j_{1}\right)$, and so on by induction: if a face $P\left(j_{1}, \ldots, j_{k}\right)$, where $j_{1}, \ldots, j_{k} \in\{1,2\}$, contains more than one vertex of $-(1 / d) S$, then there is a pair $P\left(j_{1}, \ldots, j_{k}, 1\right), P\left(j_{1}, \ldots, j_{k}, 2\right)$ of opposite facets of $P\left(j_{1}, \ldots, j_{k}\right)$, which contains all vertices of $-(1 / d) S$ lying in $P\left(j_{1}, \ldots, j_{k}\right)$.

\section{Final Remarks}

We give some remarks as requested by the referee, who asked about the motivation of the research, suggested to present a "wider picture," and asked about connections to the problem of finding large simplices in a cube.

The motivation for this research about large parallelotopes in a simplex was the necessity of an example. In [9] the following analog of the well-known theorem of John [7] about the ellipsoid of maximal volume contained in a convex body was announced. Let $C$ be a convex body and let $D$ be a centrally symmetric convex body in $E^{d}$. If $D^{\prime}$ is an affine image of $D$ of maximal possible volume contained in $C$, then $C \subset(2 d-1) D^{\prime}$. When writing [9], the author was not sure if the ratio $2 d-1$ was the best possible. Candidates, in order to show that the ratio cannot be improved, were a simplex $S$ in place of $C$, a parallelotope $P$ in place of $D$, and the parallelotope $P_{a}$ defined at the beginning of this paper as $D^{\prime}$. Since the smallest positive $k$ such that $S \subset k P_{a}$ is equal to $2 d-1$, it was sufficient to show that $P_{a}$ is a parallelotope of maximum volume in $S$. This task is performed in this paper. The theorem announced in [9] is proved in [11] which was submitted after the manuscript of the present paper was ready. 
Miscellaneous problems of maximizing the volume of an affine image of a convex body that is a subset of another convex body are considered in many papers. The pioneer seems to be Blaschke [3] who showed that every planar convex body $C$ contains a triangle of area $3 \sqrt{3} \pi^{-1} \operatorname{vol}(C)$. This estimate cannot be improved for ellipses. The analogical $d$ dimensional question remains open. McKinney [13] answered this question for the class of centrally symmetric bodies. He proved that every centrally symmetric convex body contains a simplex of volume at least $\left(1 / d ! \pi_{d}\right) \operatorname{vol}(C)$, where $\pi_{d}$ denotes the volume of the unit ball in $E^{d}$. This value cannot be lessened in the case of an ellipsoid as $C$. A classic result of this kind is the theorem of John [7], about large ellipsoids in a convex body. Macbeath [12] considered large parallelotopes in $C$. He proved that every convex body $C \subset E^{d}$ contains a parallelotope of volume at least $d^{-d} \operatorname{vol}(C)$. This estimate has been improved up to $d^{-d+1} \operatorname{vol}(C)$ in [10]. Radziszewski [14] obtained the best possible estimate $\frac{2}{9} \operatorname{vol}(C)$ for $d=3$.

Many papers consider large simplices in a parallelotope, or, equivalently, in a cube. See the survey paper of Hudelson et al. [6] and also the survey paper of Brenner and Cummings [4] about the equivalent Hadamard maximum determinant problem. The problem considered in the present paper is in a sense dual to those well-known problems. Observe that if $\Delta$ is a simplex of maximum volume in a parallelotope $P$, then $P \subset-d \Delta$. (If it were not true, then we could construct in $P$ a simplex of volume larger than the volume of $\Delta$ by moving one vertex of $\Delta$ such that it remains in $P$ but that its distance from the hyperplane containing the opposite facet increases.) Thus $\Delta \subset P \subset-d \Delta$. Denote $-d \Delta$ by $S$. Then $\Delta=-(1 / d) S$. We have $-(1 / d) S \subset P \subset S$. Consequently, Lemma 2 can be applied here. We see that every simplex of maximum possible volume contained in the unit d-dimensional cube $K=[0,1]^{d}$ contains exactly one unit segment parallel to each given axis. As a consequence, if $\Delta$ is a simplex of maximum possible volume in $K$, then for every $i \in\{1, \ldots, d\}$ the set $\rho_{i}(\Delta)$ is a prism. The base of this prism is the convex hull of the $i$ th projection of the set of all vertices of $\Delta$. The apex of this prism is in the opposite facet of $K$. Since the operation of Radziszewski does not change the volume, the volume of this prism is equal to the volume of $\Delta$. We see that the maximal volume of a $d$-simplex in $K$ is exactly $d$ times smaller than the maximum $(d-1)$-dimensional volume of a convex hull of $d+1$ vertices of $a(d-1)$-dimensional unit cube. If we take $d+1$ in place of $d$, we get the equivalent question: how large can the volume of the convex hull of $d+2$ vertices of $K$ be? It is natural to put the more general question: how large a volume can the convex hull of some $j$ vertices of $K$, where $d+1 \leq j \leq 2^{d}$, have? The case $j=d+1$ is just the question about the maximum volume of a $d$-simplex in $K$.

\section{Acknowledgment}

I would like to express my gratitude to Stanisław Szarek for valuable comments.

\section{References}

1. K. Ball: Normed Spaces with a Weak Gordon-Lewis Property in Functional Analysis, Lecture Notes in Mathematics, vol. 1470, Springer-Verlag, Berlin, 1991. 
2. A. Bielecki and K. Radziszewski: Sur les parallélépipédes inscrits dans les corps convexes, Ann. Univ. Mariae Curie-Sktodowska Sect A 7 (1954), 97-100.

3. W. Blaschke: Über affine Geometrie III: Eine Minimumeigenschaft der Ellipse, Leipzieger Ber. 69, 3-12.

4. J. Brenner and J. Cummings: The Hadamard maximum determinant problem, Amer. Math. Monthly 79 (1972), 626-630.

5. I. Fary and L. Redei: Der zentralsymmetrische Kern und die zentrallsymmetrische Hülle von konvexen Körpern, Math. Ann. 122 (1950), 205-220.

6. M. Hudelson, V. Klee, and D. Larman: Largest $j$-simplices in $d$-cubes: some relatives of the Hadamard maximum determinant problem, Linear Algebra Appl. 241-243 (1996), pp. 519-598.

7. F. John: Extremum problems with inequalities as subsidiary conditions, Courant Anniversary Volume, Interscience, New York, 1948, pp. 187-204.

8. M. Lassak: Approximation of convex bodies by parallelotopes, Bull. Polish Acad. Sci. Math. 39 (1991), 219-223.

9. M. Lassak: On the Banach-Mazur distance, J. Geom. 41 (1992), 11-12.

10. M. Lassak: Estimation of the volume of parallelotopes contained in convex bodies, Bull. Polish Acad. Math. Sci. 41 (1993), 349-353.

11. M. Lassak: Approximation of convex bodies by centrally-symmetric bodies, Geom. Dedicata, 72 (1998), 63-68.

12. A. M. Macbeath: A compactness theorem for affine equivalence-classes of convex regions, Canad. J. Math. 3 (1951), 54-61.

13. J. R. McKinney: On maximal simplices inscribed in central convex sets, Mathematika 21 (1974), 38-44.

14. K. Radziszewski: Sur une probléme extrémal relatif aux figures inscrites et circonscrites aux fiures convexes, Ann. Univ. Mariae Curie-Skłodowska Sect A 6 (1952), 5-18.

Received May 15, 1997, and in revised form December 21, 1997. 